\title{
CelestinesCa
}

https://doi.org/10.7203/Celestinesca.13.19702

INDEX TO CELESTINESCA 1-12 (1977-1988)*

\author{
Joseph T. Snow \\ University of Georgia \\ I. General Author Index \\ II. Index of Reviews \\ III. Iconographic Index
}

\section{GENERAL AUTHOR INDEX}

The General Author Index was organized to include all items which appear with independent authorship in the pages of the first twelve volumes of Celestinesca. To these I have added, from the PREGONERO sections, all conference papers for which abstracts or summaries were published (many other paper titles are included there but not listed here) and all manner of performances of celestinesque works for which sufficient information was available for summarizations. Each item appears in this index with its own rubric, following the title, as indicated: articles (A), notes $(N)$, reviews $(R)$, review-articles (RA), performance summaries (PS), necrologies (O), bibliographies (B), reprints (RPT), texts (T), translations (TR), poems $(P)$, creative works $(C)$, research reports $(R R)$, and conference reports $(C R)$. Volume, issue, and page number(s) complete the entries.

ANDERSON, Reed, rev. of Calisto and Melibea (opera), Davis, California, June 1979 (R)

$\begin{array}{rr}\text { 3:ii } & 27-30 \\ 6: \mathrm{i} & 31-32 \\ \text { 12:i } & 45-50 \\ & \\ 2: \mathrm{i} & 3-6 \\ & \\ \text { 2:ii } & 29 \\ \text { 2:ii } & 3-\mathrm{i} 1 \\ \text { 10:i } & 74\end{array}$

BALDWIN Jr, S., Sin and Retribution in the Celestina (AB)

ARMISTEAD, S. G., and J. H. Silverman, Un poema celestinesco en la tradición sefardi moderna (A)

and J. H. Silverman, Un poema celestinesco en la tradición sefardi moderna (nota adicional) (N) 29

A YERBE-CHAUX, Reinaldo, La triple tentación de Melibea (A) 74

- I would like to express my deep appreciation to Karen Coker for her sound advice, organization skills, and patience during the preparation of these indexes. 
BARRICK, Mac E., Celestina's Black Mass (N)

BEARDSLEY, Jr., Theodore S., Celestina, o los dos trabajadores: A Shadow Play of 1865 (A)

10:ii

$17-24$

, The Lowlands Editions of Celestina (1539-1601) (A)

BELTRAN, Rafael, Paralelismos en los enamoramientos de Calisto y Tirant lo Blanc: Los primeros sintomas del mal del amar (A)

BENITO de Lucas, Joaquín, Dos poemas de amor (P)

BERNDT-KELLEY, Erna, Elenco de ejemplares de ediciones tempranas del texto original y de traducciones de la obra de Fernando de Rojas en Canada, Estados Unidos y Puerto Rico (B)

Peripecias de un título: en torno al nombre de la obra de Fernando de Rojas (A)

BERSHAS, Henry N., Testigo es el cuchillo de tu abuelo (Celestina I) (A)

BERTRAND, Joseph, Theatre (Liliane Wouters, Brussels 1981)

BRUTON, Shirley, Pármeno's Search for Identity (AB)

BURKE, James F., rev. of Fernando Cantalapiedra's Lectura semiotico-formal de 'La Celestina' (R)

CANET VALLES, José Luis, La Comedia Thebayda, una 'reprobatio amoris' (A)

CANTALAPIEDRA, Fernando, Los refranes en Celestina y el problema de su autoria (N)

CARDENAS, Anthony J., The corriente talaverana and the 
CARDONA, Angeles, En torno a la edición de $L C$ nacida a raiz del Congreso Internacional de LC (AB)

CAVAllero, Pablo A., Algo más sobre el motivo grecolatino de la vieja bebedora en Celestina. Rojas y la tradición de la comediografia (A)

CORFIS, Ivy A., The Primer entremés de Selestina An Edition, with an Introduction, Notes and a Reading Text of an Anonymous Celestinesque Work of the Sixteenth Century (T) rev. of Fernando de Rojas, Celestina: Tragicomedia de Calisto y Melibea (edición y estudio crítico), ed. Miguel Marciales (RA)

10:ii $\quad 43-48$ rev. of Ciriaco Morón Arroyo's Sentido y Forma de 'La Celestina' (R)

$9: i \quad 47-48$ , Juan de la Cueva's Sonnet on Celestina (T) 7:ii $\quad 21-22$ Celestina comentada. A Case of Law and Literature (AB)

8:ii 43 La Celestina comentada y el código jurídico de Fernando de Rojas (AB)

, The Status of Women in Courtly Literature (AB)

11:ii 47 , Dark Laughter: Irony in Celestina (AB)

COSTA FONTES, Manuel da, Celestina's Hilado and Related Symbols (A)

Celestina's Hilado and Related Symbols:

A Supplement (N)

CRIADO DE VAL, Manuel, 'Amor Impervio' (LC, I, 48):

What Does It Mean? (A)

CRUZ-SAENZ, Michelle S. de, rev. of Compañia Teatro del Aire's La tragicomedia de Calisto y Melibea (R)

CZARNOCKA, Halina, Sobre el problema del espacio en Celestina (A)

DARDON TADLOCK, Gisela, rev. of Celestina (teatro) in San Francisco, California (R) 


\section{CELESTINESCA}

DE VRIES, Henk, Isaco Coeno de...¿dónde? (N)

$10: \mathrm{ii}$ 41

'Mas ygual galardón': sobre estructura y autoria de $L C$ (AB)

$10:$ ii

Cryptografie, een nijzonder geval: het boek dat Celestina wordt genemd (AB)

$10: \mathrm{ii}$

DEYERMOND, Alan, Divisiones socio-económicas, nexos sexuales: la sociedad de Celestina (A)

$\begin{array}{lr}\text { 8:ii } & 3-10 \\ \text { 1:i } & 6-12 \\ 2: i & 25-30 \\ 4: i i & 31-34 \\ & \\ 1: i & 13-18\end{array}$

La Celestina. The Art of Alvaro Custodio and Amparo Villegas (A)

DRYSDALL, D. L., The French Version of the Penitencia De Amor (A)

9:i 23-31

The Guillaume Chaudiere Edition of Jacques de Lavardin's Célestine (N)

DUBNO, Barbara Riss, and John K. Walsh, Pero Diaz de Toledo's Proverbios de Seneca and the Composition Celestina, Act IV (A)

$11: i \quad 3-12$

DUMUR, Guy, Theatre (P. Ionesco, Créteil, France 1983) (R)

$8: i$

EESLEY, Anne, Celestina's Age: Is She Forty-Eight? (A)

10:ii $25-30$ , Four Instances of '¡Confessión!' in Celestina (N) , "Carta" (response to $\mathrm{K}$. Whinnom on the use of the article in the title of Rojas' work) (T) , The Third Person in Celestina, diss. (AB)

ELLIS, Deborah, 'AAdios, paredes!' The Image of the Home in Celestina (A) 


\section{CelestinesCA}

FAULHABER, C. B., The Celestina and the Libro de buen amor (AB)

Huellas de Celestina en la Tragicomedia de Lisandro

y Roselia, de Sancho de Muñon (A)

FELIPE, Leon, "A Celestina" (P)

6:ii $\quad 35-36$

FERRE, Rosario, Celestina en el tejido de la cupiditas (A)

7:i

3-16

FERRECCIO PODESTA, Mario, 'Haba morisca', ¿haba marisca? (A)

8:ii

$11-16$

FERRER Y CHIVITE, Manuel, Unos momentos en la vida de Fernando de Rojas (A)

FINCH, Patricia S., Religion as Magic in the Tragedia Policiana (A)

, The Uses of the Aside in Celestina (A)

6:ii

, Gerarda como figura celestinesca (AB)

5:i

Witchcraft and the Concept of "Admiration in the Celestina (AB)

, Magic and Witchcraft in Celestina and Its Imitations, diss. (AB)

Magic and Moral Intent in $L C$ and Its Imitations (AB)

FORCADAS, Alberto, Debatabilidad de la teoria de la

errata de imprenta en haba morisca (A)

'Mira a Bernardo' es alusión con_sospecha (A)

Sobre las fuentes históricas de '...eclipse ay mañana',

etc., y su posible incidencia en acto I de Celestina (A)

FOTHERGILL-PAYNE, Louise, La cambiante faz de la Celestina.

Cinco adaptaciones de fines del siglo XVI (A)

, Otra perspectiva de Celestina (Adaptación

de José Blanco Gil, El Paso, 1985) (R)

FRAKER, Charles F., Declamation and the Celestina (A)

GALlO, Lee A., Celestina. A New Social Perspective (AB)

GARAY, René, El concepto de la máscara en la Celestina (A)

GARCI-GOMEZ, Miguel, 'Amor imperuio' o 'amor improuo' (LC I, 94) (A) 4:i 


\section{CELESTINESCA}

, 'Huevos asados': afrodisiaco para el marido de Celestina (A)

El sueño de Calisto (A)

9:i $\quad 11-22$

Fernando de Rojas and the Turn of Love from Courtly to

Predatory (AB)

$7: \mathrm{ii} \quad 27-28$

, On Courtly Love and the Celestina (AB)

$9:$ i $\quad 65-66$

GASCON-VERA, Elena, Celestina, Dama Filosofia (A)

$7:$ ii $\quad 3-10$

- Celestina Dialogue as Mirror of Parody (RR)

$7:$ ii $\quad 35-36$

, Celestina y el Descubrimiento (AB)

10:ii

55

GERITZ, Albert J., Calisto and Melebea (1530) (A)

$4: i \quad 17-29$

, Calisto and Melebea. A Bibliography (B)

3:ii $\quad 45-50$

GERLI, E. M., 'Mira a Bernardo': Alusión Sin Sospecha (A)

1:ii $\quad 7-10$

A Propos the Pantomime Ox, Sexual Innuendo, and

Fuddled Partridges: Yet More on Pármeno's Remark (N)

12:ii

$55-59$

GIL, Antonio C. M., Violence in the Search of Love and Honor in Celestina (AB)

8:ii

43

GULSTAD, Daniel E., Courtly Love and Rojas' Celestina (AB)

9:i

66-67

GURZA, Esperanza, rev. of Celestina, Circulo de Bellas Artes

(Madrid, 1974), Directed and Adapted by A. Facio (R)

$9: \mathrm{i}$

$56-62$

, La oralidad y la Celestina (AB)

4:ii

Orality in $L C(R R)$

4:ii

45-47

GWARA, Joseph J., rev. of Hispanic Studies in Honor of Alan D. Deyermond: A North American Tribute, ed. John S. Miletich (R)

$11: \mathrm{i}$

$41-44$

Ibid (correction)

11:ii

HANDY, Otis, The Rhetorical and Psychological Defloration of Melibea (A)

HESSE, Everett W., rev. of La Celestina (teatro) at El

Chamizal, March 1980 (R)

4:ii $\quad 39-40$

HOOK, David, 'Andar a caça de perdizes con bueyes' (N)

8:i $\quad 47-48$

, 'Fons curarum, fluvius lachrymarum': Three Variations

Upon a Petrarchan Theme (C. de Pisan, F. de Rojas and

Fray Luis de Granada) (A)

6:i $\quad 1-7$ 


\section{CELESTINESCA}

Pármeno's 'falso boezuelo' Again (N)

9:i $\quad 39-42$

John London, Clare Ludden, Amanda Taylor, and

Melanie Strickland, A Triple Review, Celestina

Directed by Christopher Fettes, Drama Centre,

London, November 1984 (R)

$9: i \quad 51-55$

JOHNSON, Julie G., Three Celestinesque Figures

of Colonial Spanish American Literature (A)

5:i $\quad 41-46$

JONES, Joseph, R., Comedia Poliscena. Introductory and

Bibliographical Notes, Text, and Translation: Part I (N, B)

9:ii $\quad 85-94$

, Comedia Poliscena per Leonardum Aretinum Congesta

(edición del texto de 1478) (T)

$10: \mathrm{i} \quad 23-44$

The Play of Poliscena, Composed by Leonardo Aretino

(traducción al inglés del texto de 1478) (TR)

$10: \mathrm{i} \quad 45-67$

JOSET, Jacques, Apuntes sobre la versión en

valón de La Celestina de Marcel Hicter (N)

12:ii $\quad 67-72$

, Una Celestina romana y francesa (teatro),

at Vaison-la-Romaine (R)

5:ii

54

, De Pármeno a Lazarillo (A)

8:ii $\quad 17-24$

4:ii

27

KASTEN, Lloyd, Mack Hendricks Singleton (1908-1980) (O)

$11: \mathrm{i}$

KENNEDY, Angus J., A Note on Christine de Pizan
and Petrarch (N)

KENNEDY, Angus J., A Note on Christine de Pizan
and Petrarch (N)

KIRBY, Steven D., Observaciones pragmáticas sobre tres aspectos de la crítica celestinesca (AB)

$12: \mathrm{i}$

KISH, Kathleen V., and Ursula Ritzenhoff, the Celestina

Phenomenon in Sixteenth-Century Germany: C. Wirsung's

Translations of 1520 and 1534 (A)

4:ii $\quad 9-18$

, On Translating 'huevos asados': Clues From

Christof Wirsung (A)

4:ii $\quad 19-31$

, Mack Hendricks Singleton (1908-1980) (O)

4:ii $\quad 29-30$

, Celestina en Amberes en el siglo XVI (AB)

$10: \mathrm{ii}$

56

, On Editing Celestina Translations: Marginalia, Rezeption, and Not So Trivial Pursuits (AB)

KULIN, Katalin, Leyendo La Celestina (A)

$4: \mathbf{i}$

$9-15$

LAW, John R., Calisto as the Antithesis of Fifteenth-Century nobleza (AB) 


\section{CELESTINESCA}

LEE, Cecilia C., Ya quiere amanecer y la plenitud del amor. Poesias de Manuel Mantero (R)

LIDA DE MALKIEL, M. R., The Earliest Trace of Euripides in Spanish Literature (N)

9:ii $\quad 75-79$

, La originalidad de La Celestina (A)

8:i $\quad 16-22$

, Carta (M.R. Lida de Malkiel a D.W. McPheeters) (T)

$11: \mathrm{i} \quad 21-23$

LIHANI, John, Spanish Urban Life in the Late Fifteenth

Century as Seen in Celestina (A)

11:ii 21-28 , rev. of La Comedia llamada Serafina, ed. Glenn F.

Dille (R)

4:ii

$35-37$

LONDON, John [see HOOK]

LOUREIRO, Angel, Calixto en el jardin de Melibea (P)

LUDDEN, Clare [see HOOK]

MADRIGAL, José A., Entrevista a José Blanco

Gil, director de La Celestina en portugués ( $R$ )

9:ii $\quad 95-101$

MALKIEL, Yakov, A Brief History of M.R. Lida's

Celestina Studies (A)

$\begin{array}{lr}\text { 6:i } & 3-13 \\ 8: \text { ii } & 15-28 \\ 2: \text { i } & 31-33 \\ 2: \text { ii } & 37-38\end{array}$

California, November 1978 (R)

McVAY, Jr., Ted E., A Possible Hidden Allusion 
MORALES, Ricardo José, ¿Tres Celestinas en

el Museo del Prado? (A)

MORGAN, Erica, Rhetorical Technique in the Persuasion of Melibea (A)

MORON-ARROYO, Ciriaco, Sobre el llamado existencialismo en La Celestina. Esperanza Gurza, Lectura existencialista de ' $L C$ ', Madrid: Gredos, 1977 (R)

MOUDOUD, Chantal Cassan, El uso de los apartes en Celestina (A)

11:i $\quad 13-20$

MUNDI PEDRET, Francisco, Elementos religiosos, positivos y negativos, en $L C$ (AB)

$10: \mathrm{ii} \quad 54-55$

MUNOZ GARRIGOS, J., Concordancias de Celestina (RR)

$7: \mathrm{ii}$

NAYLOR, Eric W., rev. of Ramon Diaz-Solis'

Tarde en Espana (R)

NODAR MANSO, Francisco, $L C$ : una expresión de las estructuras temático-narrativas de la poesia lirica cancioneril (AB)

10:ii 54

ORDUNA, Germán, Auto "Comedia " Tragicomedia " Celestina. Perspectivas críticas de un proceso de creación y recepción literaria (A)

ORTIZ GRIFFIN, J. L., The Transformations of Calisto and Melibea in $L C(\mathrm{AB})$ - The Metamorphosis of Calisto and Melibea (AB)

OSUNA LAMEYER, José, Reflexiones sobre mi puesta en escena de Celestina (N)

PENAS-BERMEJO, Francisco, El progresivo desarrollo lingũístico de Melibea como resultado de su evolución sicologica (AB)

QUEVEDO, Francisco de, "A Celestina" [Epitafio poético] (P)

$2: \mathrm{i} \quad 47-48$

"R", La Celestina; o tragi-comedia de Calixto y Melibea (1836) (RPT) , rev. of Fernando de Rojas' Celestina, ed. with introd. and notes by Dorothy $S$. Severin, with trans. of James Mabbe (1631) (R) , rev. of Peter Russell's Temas de 'La Celestina' 


\section{CELESTINESCA}

, The Genre of $L C$ (AB)

$5: \mathrm{i}$

58

, Celestina and Its 'Theatrum Vitae' of Common Wisdom (AB)

8:ii

42

RIELO PARDAL, Fernando, La dialéctica de lo antimístico en LC (AB)

$10: \mathrm{ii}$

RITZENHOFF, Ursula [See KISH]

ROUND, Nicholas G., Celestina secundum litem: Miguel Marciales' Carta a Stephen Gilman (RA)

$11: \mathrm{i} \quad 25-40$

ROZEMOND, J. J., 'Eclipse ay manana, la puente es

llevada': Dos notas sobre la fecha de Celestina (N)

6:ii

$15-18$

RUBIO, Carlos, El juego de seducciones de La Celestina: una estructura dramática (A)

SanCheZ-Gonzalez, A., La Peña Celestina (RPT)

SANTANA, Mario, Crispin vs Polichinela: Unas similitudes dramáticas entre Celestina y Los Intereses Creados de Benavente (N)

rev. of Federico Romero's Calisto y Melibea. Tragicomedia en verso, en tres actos, basada en la clásica abra de Rojas (R)

Melibea: personaje escindido en una tragedia de la transgresión (AB)

11:ii $\quad 46-47$

SCHNEIDER, J. F., rev. of H. Lopez Morales, ed., intro., y notas, La Celestina (R)

$1: \mathrm{i} \quad 21-22$

SENIFF, Dennis, Bernardo Gordonio's Lilio de Medicina.

A Possible Source of Celestina? (N)

$10: \mathrm{i} \quad 13-18$

'El falso boezuelo con su blando cencerrar': or, The

Pantomime Ox Revisited (N)

$9: \mathrm{i}$

43-45

SEVERIN, Dorothy S., Cota, His Imitator, and

La Celestina: The Evidence Reexamined (A)

'El falso boezuelo', or the Partridge and the

Pantomime Ox (N)

4:i

$31-33$

F. de Rojas and Celestina: The Author's Intention from

Comedia to Tragicomedia de Calisto y Melibea (A)

$5: i$

$1-5$

A Minimal Word-Pair Study of Celestina.

More Evidence about the Authorship of Act I (N)

$7:$ ii

$11-12$ , and Joseph T. Snow, La casa de Pleberio en Salamanca (N)

$12: \mathrm{i} \quad 55-58$ 


\section{CELESTINESCA}

Position Paper on Celestina and the Parody of the Sentimental Romance (AB)

La Celestina y el discurso novelistico (AB)

10:ii

The Celestina as Performance Text (AB)

10:ii

The Celestina's Courtly Lyrics and James Mabbe's English

Translations (AB)

10:ii

$L C$ y el discurso novelístico (AB)

10:ii

56

SHIPLEY, George A., Experience and Authority in LC (AB)

4:ii

43-44

SILVERMAN, J. H., [See ARMISTEAD]

SNOW, J. T., La Tragicomedia de Calisto y Melibea de Juan de Sedeño: Algunas observaciones a su primera escena, comparada con la original (A)

, La Celestina of Felipe Pedrell (A)

, Marcel Bataillon (1895-1977) (O)

The Iconography of the Early Celestinas (I):

The First French Translation (1527) (A)

and D.S. Severin, La Casa de Pleberio en Salamanca (N)

$12: i \quad 55-58$

, rev. of Calisto and Melebea (ca. 1530) (recording) (R)

6:ii $\quad 31-33$

rev. of The Calisto and Melibea of Edwin

Honing (libretto) (RA)

3:ii

$32-40$

rev. of La comedia llamada 'Serafina', ed. Glen F. Dille (R)

$3: \mathrm{i}$

$39-41$

rev. of Fernando de Rojas' Celestina, ed. Regula

Rohland de Langbehn (R)

Celestina de Fernando de Rojas (general title): suplemento bibliográfico (B)

$\begin{array}{cc}1: \mathrm{i} & 23-45 \\ 1: \mathrm{ii} & 39-53 \\ 2: \mathrm{i} & 35-46 \\ 2: \mathrm{ii} & 49-64 \\ 3: \mathrm{i} & 45-54 \\ 3: \mathrm{ii} & 51-55 \\ 4: \mathrm{i} & 51-58 \\ 4: \mathrm{ii} & 51-58 \\ 5: \mathrm{i} & 59-62 \\ 5: \mathrm{ii} & 57-58 \\ 6: \mathrm{i} & 47-48 \\ 6: \mathrm{ii} & 26-29 \\ 7: \mathrm{i} & 41-45 \\ 7: \mathrm{ii} & 37-42\end{array}$




\section{CELESTINESCA}

$8: i$

8:ii

$9: \mathrm{i}$

9:ii

$10: \mathrm{i}$

10:ii

$11: \mathbf{i}$

11:ii

$12: \mathrm{i}$

12:ii

$10: \mathrm{i}$

, In Memoriam Keith Whinnom (1927-1986) (O)

IX Academia Literaria Renacentista (Salamanca, 1988):

La Celestina (CR)

Celestinesca: Ten Years (N)

Mabbe's CELESTINA Revised and Staged (Sheffield, 1978) (PS)

The Bilingual Experiment in Los Angeles (Alvaro Custodio's LC, 1978) (PS)

More on Camilo Jose Cela's Celestina (Madrid 1978) (PS)

Celestina en las tablas (Alfonso Sastre, Rome 1979) (PS)

Celestina en las tablas (René Buch, El Paso Texas 1980) (PS)

, Celestina en las tablas (M. Manzaneque, Madrid 1980) (PS)

Claudina/Celestina's Role(s) in the Seduction of Pármeno (AB)

Informe sobre el acto del traslado de los restos de Fernando de Rojas

a Talavera de la Reina (CR)

, Un aspecto del arte teatral de Celestina: el caso de Claudina (AB)

Celestina on Stage (Alvaro Custodio's Eva y Don Juan, El Escorial

1981) (PS)

, Celestina on Stage (Angel Facio, USA tour 1982) (PS)

Reposición de Celestina en México (French/Garcini, 1982) (PS)

$61-63$

$49-53$

$71-77$

$103-108$

$75-79$

$59-64$

$57-65$

$49-59$

78-82

95- 103

$12: \mathbf{i}$

65-68

10:ii

$1-2$

2:ii

33-34

2:ii

$34-35$

2:ii

34-35

3:ii

41

4:i

45-46

4:ii

$4: \mathrm{i}$

4:ii

$5: i$

$6: i$

$6: i$

6:ii

25-26

Four Celestina Productions (Juan Guerrero Zamora on Spanish TV, 1983;

José Martín Recuerda's 'El carnaval de un reino' Madrid, 1983;

David Gilmore's 'The Fruits of Love,' Southampton, England, 1983;

and T. French/S. Garcini's 'Celestina,' Mexico City, 1982) (PS)

, Tres Celestinas en cinco meses en España (J. Blanco-Gil, Almagro 1984;

Angel Facio, Madrid 1984; [M. Narros, Madrid-planned]) (PS) 


\section{CELESTINESCA}

, Celestina in California (Video: H. Richmond, Berkeley 1981) (PS) 8:ii

A New Celestina (of sorts) (Alfonso Sastre, Barcelona 1985) (PS)

$9: i$

, Celestina on Stage (Philip Prowse, Glasgow 1986) (PS)

$10: i$

, Celestina on Stage (2 Semana de Erotismo, Madrid 1986) (PS)

10:ii

, El descubrimento del ser como motivo literario en Celestina (AB)

10:ii

54

, Lo que nos enseña la ilustración de las Celestinas en el siglo XVI (AB)

10:ii

Celestina on the Boards (Venezuela's Rajatabla Company, New York 1987) (PS)

A Video of Celestina (M. Sabido, Mexico 1979) (PS)

$12: \mathrm{i} . \quad 70-71$

- Celestina on Stage (Torrente Ballester/Marsillach, Madrid 1988)

(PS)

, Miguel Sabido's Film of Celestina. Updated Bawdy or Boring Bodies (AB)

Celestina en las tablas (Venezuela's Rajatabla Company, 1987-88;

Torrente Ballester/Marsillach, 1988-89) (PS)

, LC de A. Goris/H. Claus, Antwerp 1987 (PS)

12:ii $\quad 91-92$

, LC de M. Hicter, Liège 1964, 1981 (PS)

12:ii $\quad 90-91$

SOONS, Alan, rev. of La Picara Justina, ed. Bruno Damiani (R)

$8: i \quad 55-58$

STAMM, James R., Inconcinnity in the Tragicomedia, Act XIV (N)

$43-46$ Inconcinnity Pursued: The Secret of Sosia

and Related Matters (N)

9:ii $\quad 81-84$

, El 'plebérico coraçon': Melibea's heart? (A)

3:ii $\quad 3-6$

, Two Missing Persons: Claudina and Alberto (AB)

4:ii

44

, $L C$ : The End of the Debate (AB)

$5: i$

56

, On Courtly Love and Celestina (AB)

$9: i$

66 


\section{CELESTINESCA}

STERN, Charlotte, Two Early Allusions to Celestina (N)

12:ii $\quad 61-63$ , rev. of D.W. McPheeters' Estudios humanisticos sobre

la Celestina (R)

STRICKLAND, Melanie [see HOOK]

SURTZ, Ronald E., rev. of Pamela S. Brakhage's The Theology

of La lozana andaluza (R)

11:i

SUTHERLAND, Madeleine, Violence in the Celestina (AB)

10:i 73-74

TAYLOR, Amanda [see HOOK]

TELlo DIAZ, Pablo, "Carta" (el traslado de los restos de F. de Rojas a la catedral de Talavera de la Reina, 1980) (T)

THOMPSON, B. B., Misogyny and Misprint in La Celestina, Act I (A)

1:ii $\quad 21-28$

TYLER, Richard, La Celestina in the Centro de Estudios de los Siglos de Oro (N)

UMBRAL, Francisco, Calixto y Melibea (AB)

10:ii

URIARTE REBAUDI, L. N., Los plantos de Celestina (AB)

VALIS, Noël M., El triunfo de Celestina: The

Go-Between and the Penal Code of 1870 (A)

$5: \mathrm{i} \quad 35-40$

VAN BEYSTERVELDT, Antony, Courtly Love and Celestina (AB)

VETTERLING, Mary-Anne, rev. of Celestina (1979 film) (R)

VICENTE, Luis Miguel, El lamento de Pleberio: Contraste y parecido con dos lamentos en Cárcel de amor (A)

WALSH, John K., [see DUBNO]

WEST, Geoffrey, The Unseemliness of Calisto's Toothache (A) , rev. of J.T. Snow's Celestina by Fernando de Rojas: An Annotated Bibliography of World Interest, 1930-1985 (R) 


\section{CELESTINESCA}

WHINNOM, Keith, Dr. Severin, the Partridge, and the Stalking Horse (N)

$\begin{array}{lr}\text { 4:ii } & 23-25 \\ \text { 4:ii } & 19-21 \\ \text { 3:ii } & 25-26 \\ \text { 5:ii } & 51-53 \\ \text { 11:ii } & 29-35 \\ \text { 8:ii } & 43\end{array}$

\section{INDEX OF REVIEWS}

Book, text, or performance reviewed

Vol./no. Pages

ANDRADE, Marcel C., ed. La 'Celestina' (adapted for Intermediate Students). Lincolnwood, Illinois: National Textbook Co., 1987. 48 pp. illus. (E. Martí-López)

$11: \mathrm{i} \quad 50-52$

BLANCO GIL, José, director. A Celestina (El Chamizal, El Paso, Texas), March 1985 (Louise Fothergill-Payne)

$9: i \quad 63-64$ , [see MADRIGAL]

BRAKEHAGE, Pamela S. The Theology of 'La lozana andaluza'.

Potomac, Maryland: Scripta Humanistica, 1986. 77 pp.

(Ronald E. Surtz)

BUCH, René, adapt. and director. La Celestina (Berkeley, California), November 1977. (Adrienne S. Mandel)

, (El Chamizal, El Paso, Texas), March 1980.

(Everett W. Hesse)

4:ii

$39-40$

CANTALAPIEDRA, Fernando. Lectura semiotico-formal de 'La Celestina'.

Kassel: Reichenberger, 1986. Problema semiotica, 8.

229 pp. (James F. Burke)

$11: \mathrm{ii} \quad 37-39$

CUSTODIO, Alvaro, adapt. and director. La Celestina (Los Angeles,

California), October 1978. (Adrienne S. Mandel)

2:ii $\quad 37-38$

DAMIANI, Bruno, ed. La picara Justina. Potomac, Maryland:

Studia Humanitatis, 1982. 498 pp. (Alan Soons)

$8: i \quad 55-58$ 


\section{CELESTINESCA}

DIAZ-SOLIS, Ramón. Tarde en España. Bogotá: Tercer Mundo, 1980. 194 pp. (Eric W. Naylor)

DILLE, Glen F., ed. La comedia llamada 'Serafina'. Carbondale and Edwardsville: University of Southern Illinois Press, 1979. xxvii +114 pp. (J.T. Snow)

FACIO, Angel, adapt. and director. Celestina (Madrid), October 1984. (Esperanza Gurza) , (Washington, D.C.), March 1982 (Michelle S. de Cruz Saenz)

FERRERAS SAVOYE, J. La Célestine ou la crise de la société patriarcale. Paris: Ed. Hispano-Americanas, 1977. 224 pp. (Alan Deyermond)

FETTES, Christopher, director. Celestina (London), November 1984. (David Hook; John London; Clare Ludden, Melanie Strickland, and Amanda Taylor)

GURZA, Esperanza. Lectura existencialista de 'La Celestina'. BRH - Estudios y Ensayos, 257. Madrid: Gredos, 1977. 351 pp. (Ciriaco Morón Arroyo)

Hispanic Studies in Honor of Alan D. Deyermond: A North American Tribute, ed. John S. Miletich. Madison, WI: Hispanic Seminary of Medieval Studies, 1986. $324 \mathrm{pp}$. (Joseph J. Gwara)

HONIG, Edwin (libretto). Calisto and Melibea. Providence, Rhode Island: Hellcoal Press, 1972. 57 pp. (J.T. Snow)

LANGBEHN, Regula Rohland de. Estudio preliminar, notas y vocabulario. 'Celestina' por Fernando de Rojas. Clásicos Huemul, 41.

LAVILLE, Pierre, adapt. La Célestine (Vaison-la-Romaine), July 1981. (Jacques Joset)

LOPEZ MORALES, H., ed., introd. y notas. La Celestina. Madrid: Cupsa Ed., 1976. 254 pp. (J.F. Schneider)

MADRIGAL, J. A. (interview). With José Blanco Gil, Dir. of A Celestina in Portuguese (El Chamizal, El Paso, Texas), March 1985

MANTERO, Manuel. Ya quiere amanecer y la plenitud del amor. Col. Dulcinea, 3. Madrid, 1975. (Cecilia C. Lee) 


\section{CELESTINESCA}

MARCIALES, Miguel, ed. Celestina: Tragicomedia de Calisto y Melibea.

Al cuidado de B. Dutton y J.T. Snow. Illinois Medieval

Monographs, I. Urbana: University of Illinois Press, 1985.

2 vols. I: Introducción ( $x x \mathrm{ii}+372 \mathrm{pp}$ ); II: Edicion

critica $(x+306 p p)$. illus. (Ivy A. Corf is)

$10: \mathrm{ii} \quad 43-48$

MARTINEZ MILLER, Orlando. La ética judia y 'La Celestina' como

alegoria. Miami: Ed. Universal, 1978. 280 pp. (K. Whinnom)

McPHEETERS, D. W. Estudios humanisticos sobre la 'Celestina'. Potomac, Maryland: Scripta Humanistica, 1985. 107 pp.

(Charlotte Stern)

10:ii

$49-50$

MILETICH, J. S. [see Hispanic Studies]

MORON ARROYO, Ciriaco. Sentido y forma de 'La Celestina', 2nd ed.

Madrid: Cátedra, 1984. 134 pp. (Ivy A. Corfis)

$9: \mathrm{i} \quad 47-48$

RASTELL, John (ascribed). Calisto and Melibea (disc recording).

Dir. John Barton for the BBC, 1970. (J.T. Snow)

6:ii $\quad 31-33$

ROMERO, Federico, adapt. Calisto y Melibea. Tragicomedia en verso, en tres actos, basada en la clásica obra de Rojas.

Madrid: Herederos de Federico Romero, 1983. 184 pp.

(Mario Santana)

11:i $\quad 47-50$

ROSEN, Jerome (composer) and Edwin Honig (libretto). Calisto y Melibea.

Opera. The University of California at Davis. World première,

May 31, 1979. (Reed Anderson)

3:ii

$27: 30$

RUSSELL, Peter E. Temas de 'La Celestina' y otros estudios, del 'Cid' al 'Quijote'. Barcelona: Ariel, 1978.

508 pp. (J.R. Rank)

SABIDO, Miguel, adapt. and director. Celestina (film). Columbia Pictures, distributor, 1979. (Mary-Anne Vetterling)

SENIOR, Edward, and Wendell Phillips, adapt. La Celestina (San Francisco, California), December 1979.

(Gisela Dardon-Tadlock)

SEVERIN, Dorothy S., ed. Fernando de Rojas. Celestina. Edited, with introduction and notes, with translation of James Mabbe (1631). Hispanic Classics-Medieval, Warminster: Aris \& Phillips, 1987. xx +409 pp. (J.R. Rank)

SNOW, Joseph T. 'Celestina' by Fernando de Rojas: An Annotated Bibliography of World Interest. 1930-1985. Madison, Wisconsin: Hispanic Seminary of Medieval Studies, 1985. iii +123 pp. illus. (Geoffrey West) 


\section{CELESTINESCA}

\section{ICONOGRAPHIC INDEX}

1499 ¿Burgos?: Fadrique de Basilea: 1:ii, 9; 2:i, 33, 47; 2:ii, 29, 47, 64; 3:i, 44; 3:ii, 2 (fig.), 15, 30 (figs.); 4:i, 15 (figs.), 45; 4:ii, 16 (fig.), 37 (figs.); 5:i, 4 (figs.); 5:ii, 15, 28 (figs.), 38 (figs.), 47 (figs.); 6:i, 40 (figs.); 7:i, 16, 27; 8:ii, 26; 9:i, 2 (figs.), $10: i, 69$ (figs.)

1500 Toledo: Pedro Hagembach: 1:ii, 14 (Title); 4:ii, 58 (figs.); 6:i, 29; 9:i,69 (fig.); 9:ii, 4; 11:i, 46 (Title)

1501 Sevilla: Estanislao Polono: 9:ii, 4 (Title), 5 (Colophon)

1502 (?) Sevilla: J. Cromberger: 10:ii, 50 (Title)

1507 Zaragoza: J. Coci: 9:ii, 6 (remaining first and final pages)

1510 Toledo: Suc. de Hagembach: 9:ii, 7 (Title)

1511 Sevilla: J. Cromberger [1502?]: 9:ii, 8 (Title)

1513-15 Sevilla: J. Cromberger [1502?]: 9:ii, 8 (Title)

$1514 \quad$ Valencia: J. Joffre: 4:ii, 6, 25; 5:i, 29; 6:i, 14; 7:ii, 16, 24, 28; 8:ii, 10, 24; 9:i, 10 (Colophon); 9:ii, 9 (Title and last page); 10:i, 17; 11:i, 8

1515-16 Roma: M. Silber [Sevilla, 1502]: 12:i, 32 (final 2 pages)

1518 Valencia: J. Joffre: 8:ii, 2 (fig. of Rojas?); 11:i, 40 (same fig.)

1518-20 Sevilla: J. Cromberger [1502?]: 1:ii, 38; 6:ii, 9, 14 (Title), 24; 7:i, 25 (figs.); 8:ii, 14; 9:ii, 10 (Title); $11:$ ii, 59 (Title detail)

1523 Sevilla: Cromberger: 5:i, 46 (Title)

1525 Barcelona: C. Amorés: 7:i, 41 (fig.); 9:ii, 79 (Title), 108 (detail)

1526 Toledo: R. de Petras: 3:ii, 41; 8:i, 63 (Title); 9:ii, 11 (Title and Colophon)

1530 (?) Medina del Campo: 5:ii, 64 (Title); 9:ii, 11 (Title and Colophon), 80 (figs.)

1534 Venecia: E. da Sabio: 9:ii, 20 (Title)

1535 Venecia: P. de Nicolini da Sabio: 12:i, 34 (Title and 3 illus.); 12:ii, 32, 54, 60, 104

1538 Toledo: Juan de Ayala: 3:i, 41 (Title), 42; 6:i, 21; 7:i, 38 (Title) 


\section{CELESTINESCA}

1539

1545

1550

1553

1569

1575

1575

1599

1607

1622

1632

1633

1822

1840

1883

1886

1946

1947

1948

1967

1968

1970

1974

1987

Amberes: G. du Mont. 9:ii, 24 (Title)

Amberes: M. Nucio: 9:ii, 101 (Colophon)

Amberes: H. de Laet: 9:ii, 26 (Title and Colophon)

Venecia: G. Giolito de Ferrari: 9:ii, 20 (Title)

Alcalá: J. de Villanueva: 5:ii, 18 (Title); 9:ii, 13 (Title); 11:ii, 28 (Title); 12:ii, 3 (3 drawings)

Valencia: J. Navarro: $3: i, 32 ; 7: i, 37$

Alcalá: J. Lequerica: 12:i, 33 (Title and Colophon)

Amberes: Plantiniana: 8:i, 41 (Title); 9:ii, 25 (Title)

Zaragoza: C. Labayen: 9:ii, 12 (pp. 91-92), 15 (Title and 'Licencia')

Milano: I. B. Bidelo: 9:ii, 22 (Title)

Madrid: Viuda de A. Martín: 9:ii, 16 (Title)

Ruan: C. Osmont [bilingual, Fr.-Sp.]: 9:ii, 23 (Title)

Madrid: Leon Amarita: 6:i, 8 (Title); 9:ii, 34 (Title)

Barcelona: T. Gorchs: 4:i, 16, 30, 34; 8:ii, 40; 10:ii, 22

Barcelona: 10:i, 12, 18, 22, 42, 68 [erroneously labeled :1888']; 11:i, 12; 12:i, 68 (Escobar, illustrator)

Barcelona: D. Cortezo: 9:i, 32 (Title)

Valencia: Castalia: 6:ii, 46; 7:ii, 10, 34; 8:ii, 53; 9:i, 9, 22, 31, 38, 42, 70, 77 [label in error '1948']; 10:i, 21; 12:i 44 ['1948'] (José Segrelles, illustrator)

México: Leyenda: 4:ii, 26; 5:i, 22; 6:i, 37 (Miguel Prieto, illustrator)

Barcelona: Argos: 6:i, 33 (M. Humbert, illustrator)

Barcelona: Juventud: $8: \mathrm{i}, 46 ; 10: \mathrm{i}, 15 ; 10: \mathrm{ii}, 28,30,40$ (J. Azpelicueta, illustrator)

Barcelona: Marte: 8:i, 25, 58 (F. Ezquerro, illustrator)

Madrid: Aguilar [ed. for children]: 8:i, 28 (A. Jiménez-Landi Martinez, illustrator)

Madrid: Alfaguara: 8:i, 54 (Lorenzo Goñi, illustrator)

Lincolnwood, Illinois: National Textbook Company [adapted for schools]: $11: i, 52,65$ (G. Armstrong, illustrator) 


\section{CELESTINESCA}

\section{Celestina Imitations}

1530? Zaragoza? Jaime de Huete's Comedia Tesorina: 11:ii, 34; 12:ii, 63 (Title)

1530? Zaragoza? Jaime de Huete's Comedia Vidriana: 11:ii, 35; 12:ii, 50 (Title)

1536 Salamanca: P. de Castro: Feliciano de Silva's Segunda comedia de Celestina: 12:ii, 4 (printed upside down!)

1539 Toledo: H. de Santa Catalina: Gaspar Gómez de Toledo's Tercera Parte de la Tragicomedia de Celestina: 11:ii, 20 (Title illustration)

1542 Salamanca? Juan de Junta? Sancho de Muñon's Tragedia de Lisando y Roselia (y cuarta Celestina): 2:ii, 27 (Title)

1547 Medina del Campo: P. de Castro: Sebastian Fernández' Tragedia Policiana. 9:ii, 60,64 (Title); $12 ; \mathrm{i}, 50$

1554 Toledo: J. Ferrer: A. de Villegas Selvago's Comedia Selvagia: 9:ii, 102 (Title)

\section{Celestina Translations}

\section{Italian Alfonso Ordoñez, translator}

1506 Roma: E. Silber: 9:ii, 17 (Title and Colophon)

1515 Milano: V. Minuziano: 9:ii, 17 (Title and Colophon)

1519 Venecia: C. Arrivabene: 9:ii, 18 (Title and Colophon); 10:ii, 57 (figs.)

1525 Venecia: F. Caron: 9:ii, 19 (Title)

1531 Venecia: M. Sessa? 9:ii, 19 (Title)

1531 Venecia: A. Bindoni and M. Pasini: 9:ii, 19 (Title)

1543 Venecia: B. de Bendoni: 9:ii, 19 (Title)

\section{German Christoph Wirsung, translator}

1520 Augsburgo: S. Grimm and $M$. Wirsung: and

1534 Augsburgo: H. Stayner. 1:i, 10, 18, 45; 1:ii, 6, 20, 28, 32, 33; 2:i, 6, 11, 12, 23, 30; 2:ii, 9, 11, 12, 48 (two); 3:i, 10, 38; 3:ii, 6, 18, 24, 26, 40; 4:i, 8, 44, 49; 4:ii, 8, 17, 18, 21; 8:ii, 22, 54; 9:ii, 28 (Title 1520), 29 (Title 1534) (Hans Weiditz, illustrator) 


\section{CELESTINESCA}

French

1527 Paris: G. du Pré: 2:ii, 31; 5:ii, 50; 8:ii, 29 (four), 30 (four), 39; 9:i, 46; 9:ii, 21 (Title); 10:i, 80; 10:ii, 51; 11:ii, 60 (anonymous translator)

1578 Paris: N. Bonfons: 9:ii, 23 (Title) (Jacques de Lavardin, translator)

1633 Ruan: C. Osmont 9:ii, 23 (Title)

Dutch

1580 Amberes: Heyndric Heyndricxz: 7:ii, 26 (drawing)

English

1631 London: R. Allot 9:ii, 27 (Title); 10:i, 44 (Title) (James Mabbe, translator)

1631 London: R. Mabbe: 11:ii, 48 (Title) (James Mabbe, translator)

1973 London: Folio Society: 10:ii, 65, 66 (J. M. Cohen, translator; Dodie Masterman, illustrator)

\section{Hungarian}

1979 Budapest: Europa: 5:i, 6, 34; 5:ii, 53; 6:i, 7; 6:ii, 34 (Karoly Sandor, translator; Gyula Feledy, illustrator)

\section{'Celestina' Stagings}

1900 Title page of a comic piece, La Celestina [in Catalan], by Frederich Fuentes, fill: $12: \mathrm{i}, 53$

1949 Margarita Xirgu as Celestina, Montevideo: 12:ii, 106

1949 The Players. Performance of LC by the Univ. of Chile's Teatro Experimental: 8:i, 64

1957 Program cover. Dramatic reading of LC from M. H. Singleton's English translation, Madison, Wisconsin, Sept. 8-10: 10:ii, 58

1959 Book cover (fig. of Celestina). Celestina, adap. L. Escobar \& H. Pérez da la Ossa: 3:ii, 55

1977 The Players. Performance of LC by the Teatro Repertorio Español de Nueva York (in Berkeley, California, November 21-22): 6:i, 28

1977 The Players. Performance of LC by Teatro de la Univ. de Chile-Antofagasta: 9:i, 62 


\section{CELESTINESCA}

1978 Poster. Bilingual LC, adap. Alvaro Custodio in Los Angeles, October 6 to November 12: $3: i, 36$

1979 Poster. Calisto \& Melibea (Opera), premiere at Univ. of California at Davis (May-June): 3:ii, 31

1979 Poster. English LC of Edward Senior, San Francisco (November): 4:ii, 50

1980 Poster. Spanish LC, adap. Ricardo López Aranda, Madrid (February): 4:i, 50

1981 Ofelia González as Celestina. New York (Teatro Repertorio Español): 5:ii, 48

1981 The Players. Performance of LC of Grupo 'Teatro Joven' (Univ. of Chile, Santiago): 8:i, 39

1981 Costume sketch for Melibea. Walloon production of LC (L'acopleûse), adap.

M. Hicter: 12:ii, 64

1981 Jenny D'Inverno as Celestina. Walloon production of LC (L'acopleûse), adap. M. Hicter: 12:ii, 72

1982 The Players. Performance of LC by the Teatro de la Nacion del I.M.S.S., Mexico City (July): 7:i, 49

1982 Title. Script of a LC adaptation, "Tragedia fantástica de la gitana Celestina," by Alfonso Sastre [from Primer acto]: 8:i, 42

1983 Program cover. English adap., "The Fruits of Love," of LC, Southampton, England (February): 7:ii, 22

1984 The Players. Performance of the Portuguese LC of Lisbon's Teatro Iberico, Almagro, Spain (September): 8:ii, 44

1984 The Players. Performance of a Spanish LC by the Teatro del Aire, adap. A. Facio, Madrid (November-December): 8:ii, 48

1987 Program detail. Performance of the Dutch adap. of H. Claus, "De Spaanse Hoer, " (December): 12:ii, 103

\section{'Celestina'-related Texts}

1478 Shussenreid: Premonstratensian Monastery. Incipit of the Latin text of L. Aretino, Comedia Poliscena. 10:i, 67

1525 'Proceso' against A. de Montalbán, by the Inquisition Court of Toledo (detail): 9:ii,

1530? Calisto \& Melebea [anon. English partial translation]: 4:i, 27 (incipit); 29 (first 18 lines); 9:ii, 77 (incipit)

1540 Last will and testament of Fernando de Rojas (fragments): 10:ii, 42, 64

1584 'Limpieza de sangre' document of Hernando de Roxas: 9:ii, 32 


\section{CELESTINESCA}

1616 Title page. A. G. de Salas Barbadillo's La hija de Celestina (Milan): 4:ii, 22

1937 Text of the sales description for Venice: G. A. e P. da Nicolini da Sabio, 1541: $7: \mathrm{ii}, 42$

1949 Autograph page (copy) of a Buenos Aires presentation by Maria Rosa Lida de Malkiel: 8:i, 14

1958 Cover of LC: TCM, English translation of Mack H. Singleton (Madison: Wisconsin, UW Press): 4:ii, 28

1968 Announcements of the La Celestina of Alvaro Custodio, from Notas y Comentarios (February): 6:ii, 44

1970 Notes covering the 1969 C. Ardavin film of LC: $7: \mathrm{i}, 50$

1973 Title page of Razón y pasión de enamorados, an LC adaptation by Fernando de ToroGarland (Madrid): 12:i, 77

1980 Report on the re-burial of the remains of F. de Rojas: 4:ii, 49; 5:ii, 32

1981 Text of a play review of a French LC, Brussels (January-February): 5:i, 55

1983 Title page of Miguel Marciales' Sobre problemas rojanos y celestinescos: 11:i, 36

1985 Advertisement for the 2-vol. Estudio \& Edicion Critica of LC by Miguel Marciales (Univ. of Illinois Press): [111]; 20:i, 81

1985 Advertisement for J. T. Snow's 1985 'Celestina' by Fernando de Rojas (Hispanic Seminary of Medieval Studies): 9:ii, 78; 9:ii, 46

1987 Letter about Celestinesca from Henry King Stanford: 11:ii, 62

1987 Listing (Univ. of Pennsylvania Press) for M.E. Barrick's ed. of the Tercera Parte de la Tragicomedia de Celestina (1973): 12:i, 64

1988 Cambridge Univ. Press press release for Louise Fothergill-Payne's Seneca and 'Celestina' (1988): 12:i, 54

\section{Miscellaneous Illustrations}

1460 Engraving (celestinesque scene) by Master E. S. : 11:i, 44

1488 . Martin Vázquez de Arce [el Doncel de Sigúenza]: 5:ii, 42

1520 Burgos: Alonso de Melgar. A Celestina woodcut reused for a later 'pliego suelto': 8:ii, 26

1530 Strasbourg. Illustration by Hans Weiditz from a Herbarum: 5:ii, 30, 31

1582 - Sevilla. Illus. from G. Argote de Molina's "Discurso sobre el Libro de la monteria (the 'pantomime ox'): 9:i, 44 


\section{CELESTINESCA}

1587 Barcelona: H. Gotard. Title (figs.) used in Silva de varios romances: 9:ii, 80

1592 Illustration from Christoph Wirsung's Arzneibuch: 5:ii, 29

1601 Rome. Illus. from A. Valli da Todi's Il canto de gl'augelli (the 'pantomime ox'): $4: \mathrm{i}, 32 ; 4: \mathrm{ii}, 23$

1760? A celestinesque etching by B. Audran: 12:i, 8

1800? A celestinesque painting by Francisco de Goya: $6: \mathrm{i}, 38$

1836 Celestinesque figure, from El semanario pintoresco: $5: \mathrm{i}, 52$

1851 Illustration for Estébanez Calderon's "La Celestina": 5:i, 39 (illustrator, Giménez)

1865 Palma de Santa Catalina: J. Llorens. Illustration for "Celestina, o los dos trabajadores": 10:i, 18, 20 (incipit)

18?? A celestinesque figure, painted by Cecilio Pla: $6: i, 34$

1953 Lithograph of Amparo Villegas (as Celestina) by Vlady, for the 1953 Mexico City production of LC directed by Alvaro Custodio: 11:ii, 40

1963 "Calisto despeñado." Figure from the cover of Taurus' Teatro mdieval (ed. M. Criado de $\mathrm{Val})$ : 6:i, 19

1960s Drawings for LC by Pablo Picasso: 10:i, 16; 12:ii, 59, 93, 94 (two)

1981 Photos of a Celestina (doll) by K. W. Wolfe: 5:i, 5, 12 (two), 18, 40 (two)

1981 Lottery ticket with 'Fernando de Rojas': 5:i, 62

1982 Drawing. Plaque from Burgos' Meson del Cid, supposed locale of the first printing of the Comedia de Calisto \& Melibea: 6:i, 30

1988 Drawings. Salamanca. The "casa de Pleberio" by J. T. Snow: 12:i, 57, 59 (three)

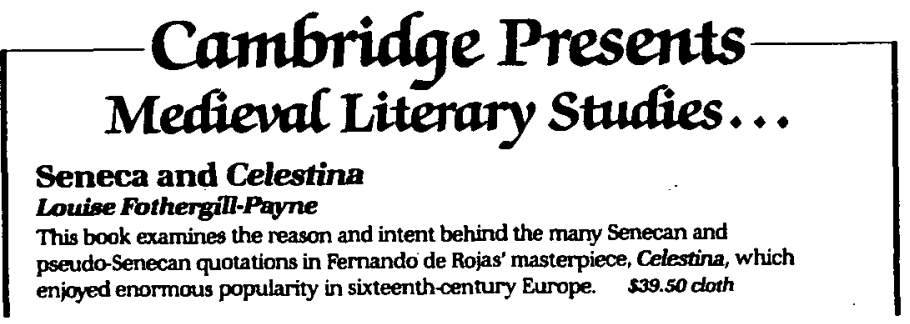




\section{CELESTINA}

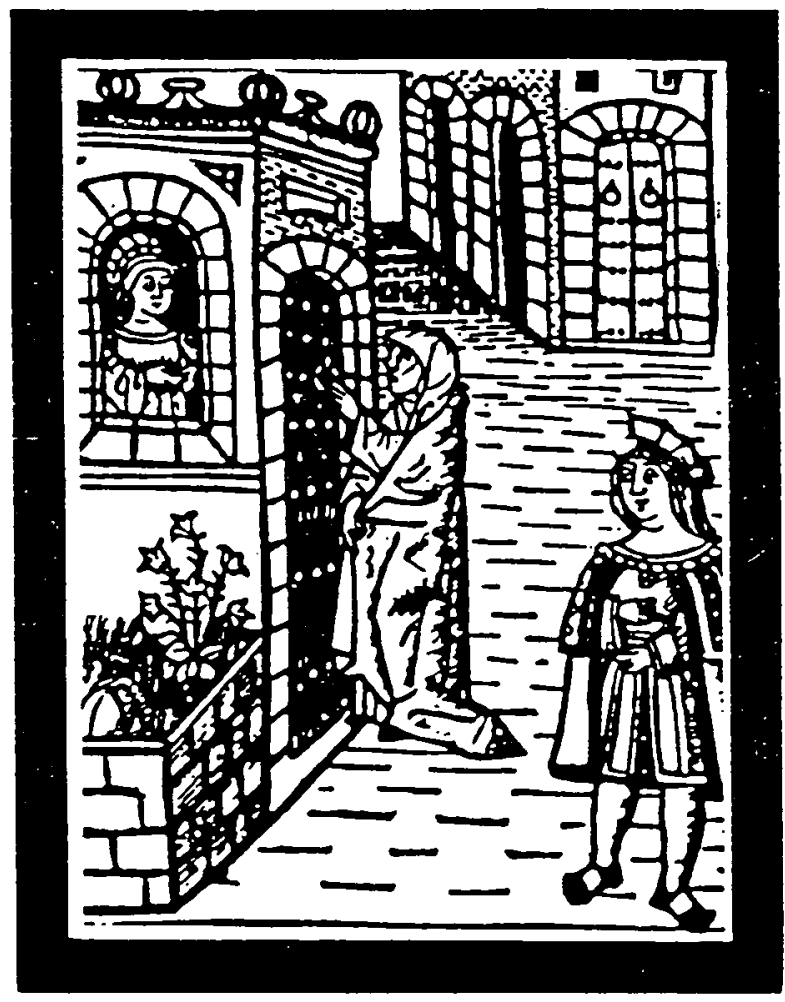

Fernando de Rojas

Edited with an introduction and notes by

Dorothy Sherman Severin

With the translation of James Mabbe (1631)

Edicion Bilingüe [1987]: Aris \& Phillips, Inglaterra 